\title{
Order parameter effect critical fields and current of $Y_{1-x} \operatorname{Pr}_{x}: 123$ superconductors
}

\author{
A. SEDKY* , S.A. AMIN \\ Physics Department, Faculty of Science, Assiut University, 71516 Assiut, Egypt
}

\begin{abstract}
Fluctuation induced conductivity by $\operatorname{Pr}$ substitution at $\mathrm{Y}$ sites of $\mathrm{Y}_{1-\mathrm{x}} \operatorname{Pr}_{\mathrm{x}}: 123$ superconductors is reported. It is found that the mean field temperature $\mathrm{T}_{\mathrm{c}}{ }^{\mathrm{mf}}$, deduced from the peak of $\mathrm{d} \rho / \mathrm{dT}$ versus $\mathrm{T}$ plot, gradually decreases by increasing Pr up to 0.40 . The order parameter dimensionality (OPD) is estimated from the slope of the logarithmic plot between excess conductivity $\Delta \sigma$ and reduced temperature $\epsilon$. Interestingly, the crossover from $2 \mathrm{D}$ to $3 \mathrm{D}$ is obtained for samples with $\operatorname{Pr}=0.00,0.10$ and 0.20 , while with increasing Pr up to 0.40 , the crossover from 0D to quasi-2D is obtained. On the other hand, the calculated values of interlayer coupling, coherence lengths, critical fields and critical current decrease with increasing $\operatorname{Pr}$ up to 0.20 , but with the further increase of Pr, up to 0.40 , they increase. The hole carriers/Cu ions anisotropy and G-L parameter gradually increase with Pr up to 0.40 . Our results are discussed in terms of the effects of Pr substitution at Y site, such as oxygen rearrangements, anisotropy, hybridization and localization of holes in the overdoped region.
\end{abstract}

Keywords: fluctuation; order parameters; excess conductivity; hole carriers

\section{Introduction}

Due to short coherence length and high value of critical temperature $T_{c}$ in high-temperature superconductors, thermal fluctuations of superconducting order parameter have been earlier observed on the conductivity versus temperature curves as excess conductivity [1]. By decreasing the temperature from room temperature toward the critical temperature $T_{c}$, the fluctuating of Cooper pairs begins to be created spontaneously at a temperature $\mathrm{T} \geqslant 2 \mathrm{~T}_{\mathrm{c}}^{\mathrm{mf}}[2-5]$. As the temperature approaches $\mathrm{T}_{\mathrm{c}}$, the number of Cooper pairs increases while the normal electron density decreases. Therefore, the resistivity decreases and the thermal fluctuations induce an excess conductivity $\Delta \sigma$ [6-8]. Therefore, resistivity versus temperature measurements in superconducting samples has been earlier explored to determine how the conductivity is affected by fluctuations of small regions of a sample in a superconducting state.

The excess conductivity analysis reveals that the contribution is due to Gaussian fluctuations in

*E-mail: sedky1960@yahoo.com the mean field and critical fluctuations regions [4]. Gaussian fluctuations are dominant in the temperature region above the mean field region when the fluctuations in the order parameters are small and the interactions between them can be neglected. The critical fluctuation occurs in the mean field region when the fluctuations in the order parameters are large and the interactions between them cannot be neglected. The variation of Gaussian fluctuations of induced conductivity with the reduced temperature $\epsilon$ helps one to find the dimensional exponents, coherence length, crossover temperatures, critical fields and critical currents of hightemperature superconductors. Fluctuations study in high- $\mathrm{T}_{\mathrm{c}}$ materials reveals that the dimension exponents are zero dimensional (0D), one dimensional (1D), two dimensional (2D) and three dimensional (3D). It has been observed that the dimensional crossover takes place between two different dimensions above $\mathrm{T}_{\mathrm{c}}$ at a crossover temperature which is different for different samples [9-13].

The disorder in high $\mathrm{T}_{\mathrm{c}}$ superconductors usually influences the critical temperature which can be verified experimentally by magnetic and nonmagnetic doping elements. It is well known that 
the fluctuation-induced conductivity is influenced by the concentration of doping elements due to the defects in the $\mathrm{CuO}_{2}$ planes of these materials and the irradiation effect [11]. If the $\mathrm{CuO}_{2}$ planes are distorted from their original structures responsible for superconductivity then the pairing conditions in the superconductor will be affected, and consequently, the fluctuation induced conductivity will be varied.

We have studied the effects of Pr substitution at $\mathrm{Y}$ sites on the structure and superconductivity of $\mathrm{Y}_{1-\mathrm{x}} \mathrm{Pr}_{\mathrm{x}} \mathrm{Ba}_{2} \mathrm{Cu}_{3} \mathrm{O}_{7}$ superconductors with various $x(0.00 \leqslant x \leqslant 0.50)$ [14]. We have also considered here the fluctuation induced excess conductivity produced by Pr substitution on the same batch of samples. The substitution by $\operatorname{Pr}$ at $\mathrm{Y}$ sites is expected to affect fluctuations through a change in oxygen disorder and anisotropy of these materials. We have restricted our analysis to the mean field regime and the dimensional crossover behavior. We also tried to extract the interlayer coupling, dimensional exponents, coherence lengths and anisotropy for such type of samples [14-16]. Furthermore, some important parameters such as critical fields and critical current were obtained. In addition, the hole carriers were calculated by two different methods to study their impact on excess conductivity.

\section{Theoretical background}

The excess conductivity $\Delta \sigma$ due to thermal fluctuation is defined as the deviation of measured conductivity of $\sigma_{\mathrm{m}}(\mathrm{T})$ from the normal conductivity $\sigma_{\mathrm{n}}(\mathrm{T})$ as follows:

$$
\Delta \sigma=\left(\frac{1}{\rho_{m}}-\frac{1}{\rho_{n}}\right)=\sigma_{m}-\sigma_{n}
$$

where $\rho_{\mathrm{m}}$ and $\rho_{\mathrm{n}}$ are the measured and normal resistivity, respectively. $\rho_{\mathrm{n}}$ is obtained from the measured resistivity $\rho_{\mathrm{m}}$ at $\mathrm{T} \geqslant 2 \mathrm{~T}_{\mathrm{c}}$ by applying the least square method to the Anderson and Zou relation [17]:

$$
\rho_{n}(T)=A+B T
$$

In order to estimate the paraconductivity, Aslamazov and Larkin (AL) deduced the following relation for the fluctuation-induced excess conductivity $\Delta \sigma$ as [18]:

$$
\Delta \sigma=A \varepsilon^{-\lambda}
$$

Here, $\mathrm{A}=\frac{\mathrm{e}^{2}}{32 \hbar \xi_{\mathrm{c}}(0)}$ for $3 \mathrm{D}, \mathrm{A}=\frac{\mathrm{e}^{2}}{16 \hbar d}$ for $2 \mathrm{D}$, $A=\frac{e^{2} \xi_{c}(0)}{32 \hbar S}$ for $1 D$, e is the electronic charge, $d$ is the interlayer spacing between two successive $\mathrm{CuO}_{2}$ planes, $\hbar$ is the reduced Planck constant, $\xi_{c}(0)$ is the c-axis 3D coherence length at zero temperature, $S$ is the wire cross-sectional area of the 1D system, $\lambda$ is an exponent of dimensionality, and their values are $0.5,1$ and 1.5 and 2 for $3 \mathrm{D}, 2 \mathrm{D}$, $1 \mathrm{D}$ and $0 \mathrm{D}$ fluctuations respectively, and $\epsilon$ is the reduced temperature given by [15-18]:

$$
\varepsilon=\frac{T-T_{c}^{m f}}{T_{c}^{m f}}
$$

where $T_{c}^{m f}$ is the mean field temperature above which the interactions between Cooper pairs can be neglected and the onset of superconducting phase coherence occurs.

We have drawn the $d \rho / d T$ versus $\mathrm{T}$ plot to obtain the values of $T_{c}^{m f}$ from the peaks. However, for polycrystalline samples, the modified equations for 2D and 3D fluctuations are expressed as [18]:

$$
\Delta \sigma_{3 D}=\frac{e^{2}}{32 \hbar \xi_{p}(0)} \varepsilon^{-\frac{1}{2}}
$$

$\Delta \sigma_{2 D}=\frac{1}{4}\left[\frac{e^{2}}{16 \hbar d} \varepsilon^{-1}\left[1+\left(1+\frac{8 \xi_{c}^{4}(0)}{d^{2} \xi_{a b}^{2}(0)} \varepsilon^{-1}\right)^{\frac{1}{2}}\right]\right]$

where $\xi_{\mathrm{ab}}(0)$ is the coherence length at $0 \mathrm{~K}$ across the ab-plane and $\xi_{p}(0)$ is the effective characteristic coherence length at $0 \mathrm{~K}$. On the other hand, the crossover behavior from 2D-3D occurs at a temperature $\mathrm{T}_{0}$ given by $[17,18]$ :

$$
T_{0}=T_{c}^{m f} \exp \left(\frac{2 \xi_{c}(0)}{d}\right)^{2}
$$

where $\xi_{c}(0)$ is given by $[19,20]$ :

$$
\xi_{c}(0)=\left(\frac{d J^{\frac{1}{2}}}{2}\right)
$$


where $\mathrm{d}=\mathrm{c}$ parameter $=11.68 \AA$ for this type of samples [21], and $\mathbf{J}$ is the interlayer coupling expressed by [22]:

$$
J=\ln \left(\frac{T_{0}}{2 T_{c}^{m f}}\right)
$$

For polycrystalline samples, $\xi_{p}(0)$ given by [23]:

$$
\frac{1}{\xi_{p}(0)}=\frac{1}{4}\left[\frac{1}{\xi_{c}(0)}+\left(\frac{1}{\xi_{c}^{2}(0)}+\frac{8}{\xi_{a b}^{2}(0)}\right)^{1} 2\right]
$$

where $\xi_{a b}(0)$ is calculated with the help of $\xi_{p}(0)$ and $\xi_{\mathrm{c}}(0)$ values. Then, the anisotropy parameter could be obtained using the relation:

$$
\gamma=\left[\xi_{a b}(0) / \xi_{c}(0)\right]
$$

The upper critical fields along the c-axis and a-b plane, and critical current density at $0 \mathrm{~K}, \mathrm{~J}(0)$, are estimated by the following relations [24, 25]:

$$
\begin{gathered}
B_{I I}(c)=\frac{\phi_{0}}{2 \pi \xi_{a b}^{2}(0)} \\
B_{I I}(a b)=\frac{\phi_{0}}{2 \pi \xi_{c}(0) \xi_{a b}(0)} \\
J_{c}(0)=\frac{2 \phi_{0}}{\sqrt{6} \pi \lambda^{2}(0) \xi_{p}(0)}
\end{gathered}
$$

where $\phi_{0}$ is a quantum flux given by $\phi_{0}=\frac{\mathrm{h}}{2 \mathrm{e}}=$ $2.07 \times 10^{-15}(\mathrm{~Wb})$, and $\lambda^{2}(0)$ is London penetration depth at $0 \mathrm{~K}$ which is about $150 \mathrm{~nm}$ for $\mathrm{R}: 123$ superconductors [26].

\section{Results and discussion}

The resistivity versus temperature curves of $\mathrm{Y}_{1-\mathrm{x}} \mathrm{Pr}_{\mathrm{x}} \mathrm{Ba}_{2} \mathrm{Cu}_{3} \mathrm{O}_{7}$ samples is given in Fig. 1. It is clear that the normal state resistivity $\rho_{\mathrm{n}}(\mathrm{T})$ is linear as the temperature is reduced from room temperature down to a certain temperature $T_{B}$. In this region $\rho_{\mathrm{n}}(\mathrm{T})$ follows the formula, $\rho_{\mathrm{n}}(\mathrm{T})=\mathrm{A}+\mathrm{BT}$ as discussed above. $\mathrm{T}_{\mathrm{B}} \simeq 2 \mathrm{~T}_{\mathrm{c}}^{\mathrm{mf}}$ is defined as the temperature below which the Cooper pair formation starts [26, 27].

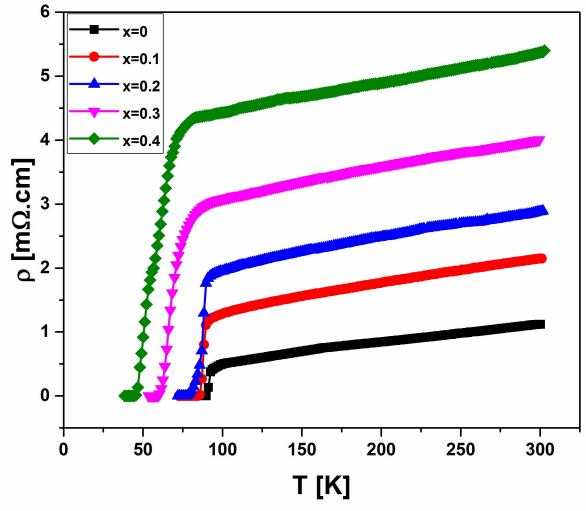

Fig. 1. Resistivity versus temperature for $\mathrm{Y}_{1-\mathrm{x}} \mathrm{Pr}_{\mathrm{x}} \mathrm{Ba}_{2} \mathrm{Cu}_{3} \mathrm{O}_{7}$ samples.

As the temperature is further reduced beyond the normal state region, the rate of change of resistivity becomes entirely different. This is mainly due to increasing Cooper pair formation as the temperature is reduced. Therefore, the fluctuation induced conductivity in this region follows the A-L model to yield the dimensional exponent appropriate to fluctuation-induced conductivity. As seen from the resistivity curves, the resistivity increases with increasing Pr content, which suggests a weakening of the metallic behavior as Pr content increases.

The normal resistivity $\rho_{\mathrm{n}}(\mathrm{T})$ is calculated in terms of $\mathrm{A}$ and $\mathrm{B}$ parameters which are obtained from the linear fit of the measured resistivity shown in Fig. 2. The mean field temperatures $\mathrm{T}_{\mathrm{c}}^{\mathrm{mf}}$ for all samples were estimated from the peak of $d \rho / d T$ against temperature plot shown in Fig. 3. After that, we calculated the excess conductivity $\Delta \sigma$ and reduced temperature $\epsilon$. Then, we plotted $\ln \Delta \sigma$ against $\ln \epsilon$ for all samples as shown in Fig. 4. We have used the general fitting of the excess conductivity with A-L equation for the concerned temperature range. It is evident from the fitting that there is a distinct difference in the slope of each plot. The corresponding temperature where the slope change occurs is designated as the crossover temperature $\mathrm{T}_{0}$. Different values of the crossover temperatures along with two different exponents have been obtained from each plot with accuracy of $\pm 1 \mathrm{~K}$ for each one. Fig. 5a shows different values of $\mathrm{T}_{\mathrm{c}}, \mathrm{T}_{\mathrm{c}}^{\mathrm{mf}}$ and $\mathrm{T}_{0}$ for all samples, and similar values are listed 
in Table 1. It is clear that both $\mathrm{T}_{\mathrm{c}}^{\mathrm{mf}}, \mathrm{T}_{0}$ are shifted to lower temperatures only in the overdoped region, at higher values of Pr content (0.30 and 0.40), in agreement with $T_{c}$ behavior. From the values of $T_{0}$ and $\mathrm{d}$ for $\mathrm{Y}: 123$ superconductors, the values of coherence lengths have been obtained and shown in Fig. 5b. Similar values are listed in Table 1.

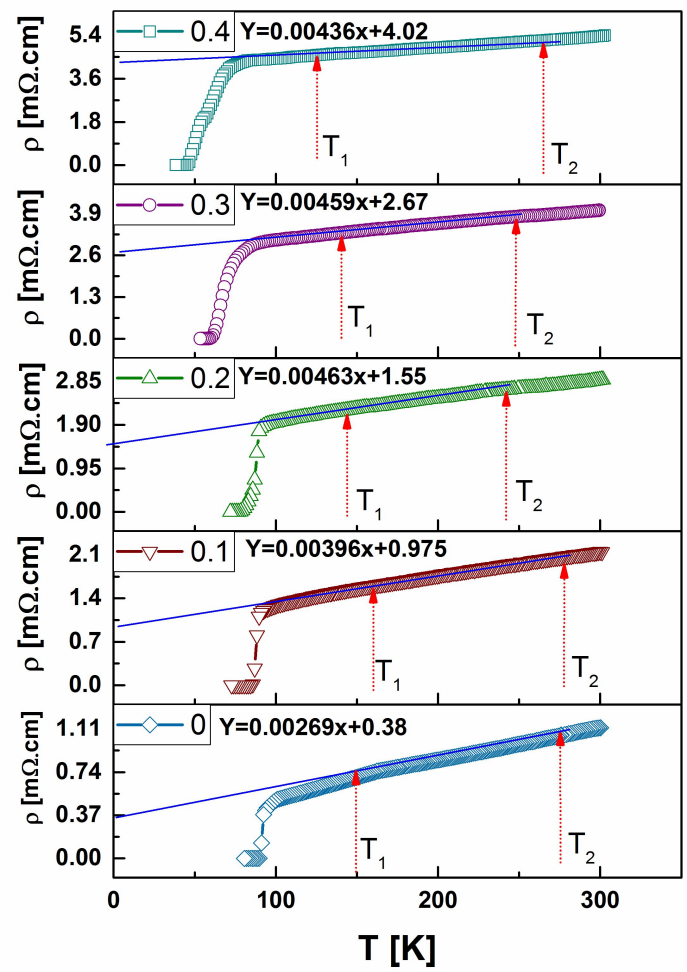

Fig. 2. Resistivity versus temperature for $\mathrm{Y}_{1-\mathrm{x}} \mathrm{Pr}_{\mathrm{x}}: 123$ samples.

In order to compare the experimental data with the theoretical predictions, different regions of the plots shown in Fig. 4 have been linearly fitted, and the values of conductivity exponent $\lambda$ have been determined from the slopes. Above $\mathrm{T}_{\mathrm{c}}{ }^{\mathrm{mf}}$, the GL theory breaks and the short-wave fluctuations play a dominant role [28]. Also, in this temperature region, the excess conductivity decreases sharply which agrees well with the theoretical prediction. With decreasing temperature towards the mean field region, an excess conductivity is obtained, and a crossover behavior is clearly observed.

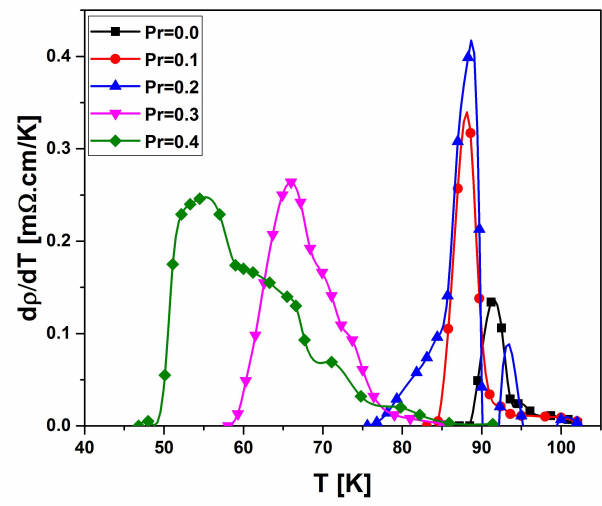

Fig. 3. $\mathrm{d} \rho / \mathrm{dT}$ versus temperature for $\mathrm{Y}_{1-\mathrm{x}} \mathrm{Pr}_{\mathrm{x}}: 123$ samples.

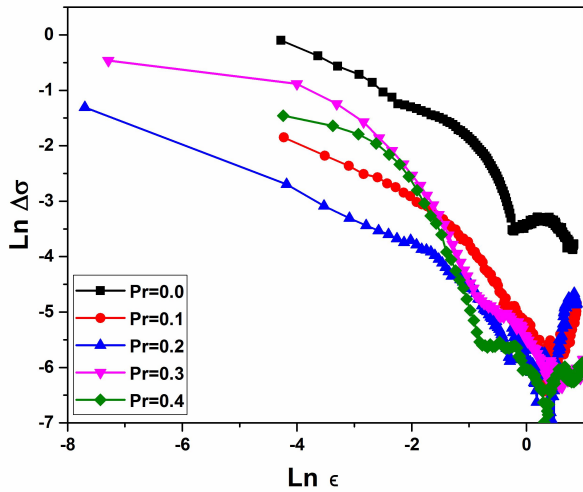

Fig. 4. $\operatorname{Ln} \Delta \sigma$ as a function of $\ln \epsilon$ for $\mathrm{Y}_{1-\mathrm{x}} \operatorname{Pr}_{\mathrm{X}}: 123 \mathrm{sam}-$ ples.

However, the slopes of the two linear regions below and above the crossover temperatures give the following dimensional exponents of these two regions: the first exponent is obtained at higher temperature in the normal field region, in which the order parameter dimensionalities (OPD) are 2D, and their values are close to $1(1.1,1.1$ and 1.05 for $\operatorname{Pr}=0,0.10$ and 0.20). With increasing Pr above 0.20 , the dimensional exponents become 1.91 and 2.26 for $\operatorname{Pr}=0.30$ and 0.40 and the OPD is OD. The second exponent is obtained at a lower temperature in the mean field region, in which the OPD are $3 \mathrm{D}$, and their values are close to $0.5(0.52,0.47$ and 0.44 for $\operatorname{Pr}=0,0.10$ and 0.20 ). With increasing Pr above 0.20 , the exponents become 0.73 and 0.77 
Table 1. $\mathrm{T}_{\mathrm{c}}, \mathrm{T}_{\mathrm{c}}^{\mathrm{mf}}, \mathrm{T}_{0},\left(\mathrm{~T}_{0} / \mathrm{T}_{\mathrm{c}} \mathrm{mf}\right), \lambda_{1}, \lambda_{2}, \mathrm{~J}, \xi_{c}(0), \xi_{\mathrm{p}}(0), \xi_{\mathrm{ab}}(0), \gamma$ and $\left(2 \xi_{\mathrm{c}}(0) / \mathrm{d}\right)$ of $\mathrm{Y}_{1-\mathrm{x}} \operatorname{Pr}_{\mathrm{x}}: 123$ samples.

\begin{tabular}{|c|c|c|c|c|c|c|c|c|c|c|c|c|}
\hline $\mathrm{x}$ & $\begin{array}{c}\mathrm{T}_{\mathrm{c}} \\
{[\mathrm{K}]} \\
\end{array}$ & $\begin{array}{l}\mathrm{T}_{\mathrm{c}}^{\mathrm{mf}} \\
{[\mathrm{K}]}\end{array}$ & $\begin{array}{c}\mathrm{T}_{0} \\
{[\mathrm{~K}]}\end{array}$ & $\mathrm{T}_{0} / \mathrm{T}_{\mathrm{c}}^{\mathrm{mf}}$ & $\begin{array}{c}\lambda_{1} \\
(2 \mathrm{D} / 0 \mathrm{D}) \\
\end{array}$ & $\begin{array}{c}\lambda_{2} \\
(3 \mathrm{D} / 2 \mathrm{D}) \\
\end{array}$ & $\mathrm{J}$ & $\begin{array}{c}\xi_{c}(0) \\
{[\AA]}\end{array}$ & $\begin{array}{l}\xi_{\mathrm{p}}(0) \\
{[\AA]} \\
{[\AA]}\end{array}$ & $\begin{array}{c}\xi_{\mathrm{ab}}(0) \\
{[\AA]}\end{array}$ & $\gamma$ & $2 \xi_{\mathrm{c}}(0) / \mathrm{d}$ \\
\hline 0.00 & 89.8 & 91.2 & 112.6 & 1.24 & 1.10 & 0.52 & 0.62 & 4.59 & 5.72 & 6.59 & 1.43 & 0.78 \\
\hline 0.10 & 84.5 & 88.4 & 106.6 & 1.21 & 1.10 & 0.47 & 0.60 & 4.52 & 5.86 & 6.99 & 1.55 & 0.77 \\
\hline 0.20 & 76.6 & 88.3 & 100.1 & 1.13 & 1.05 & 0.44 & 0.58 & 4.44 & 6.14 & 7.82 & 1.76 & 0.76 \\
\hline 0.30 & 59.3 & 65 & 76 & 1.17 & $\begin{array}{l}1.91 \\
0 \mathrm{D}\end{array}$ & $\begin{array}{c}0.73 \\
\text { Quasi-2D }\end{array}$ & 0.56 & 4.37 & 6.08 & 7.79 & 1.78 & 0.75 \\
\hline 0.40 & 44.3 & 56 & 68 & 1.21 & $\begin{array}{l}2.26 \\
0 \mathrm{D}\end{array}$ & $\begin{array}{c}0.77 \\
\text { Quasi-2D }\end{array}$ & 0.54 & 4.25 & 5.97 & 7.69 & 1.81 & 0.73 \\
\hline
\end{tabular}

for $\operatorname{Pr}=0.30$ and 0.40 , and the OPD are quasi-1D. Fig. 5c shows the behavior of OPD, J and $\gamma$ against Pr content and similar values are listed in Table 1. It is clear that OPD and $\gamma$ are gradually shifted to higher values as $\operatorname{Pr}$ increases $(\operatorname{Pr}>0.20)$, while $\mathrm{J}$ is decreased.

In their theory, Lawrence and Doniach assumed that $\mathrm{d}=11.7 \AA=$ c-parameter, and it approximately equals the spacing between superconducting $\mathrm{Cu}-\mathrm{O}_{2}$ planes. In the present case, we found that $2\left(\xi_{c}(0)\right) / \mathrm{d}=0.75$, which is twice larger than the reported values $(0.30$ and 0.54$)$ [29, 30]. Anyhow, the crossover to 3D fluctuations of Y:123 was earlier obtained at a temperature $T_{0}$ above $T$, with $\mathrm{T}_{\mathrm{o}} / \mathrm{T}=1.1$ and $1.01[28,29]$. However, our values of $\mathrm{T}_{\mathrm{o}} / \mathrm{T}$ listed in Table 1 slightly decrease with $\mathrm{Pr}$ from $1.24,1.21$ to 1.13 for $\operatorname{Pr}=0,0.10$ and 0.20. In these samples, the slope of the first normal field region is $2 \mathrm{D}$, but it is changed to $3 \mathrm{D}$ in the mean field region. With further increase of $\operatorname{Pr}$ above $0.20, \mathrm{~T}_{\mathrm{o}} / \mathrm{T}$ increased again to 1.17 and 1.21. In these samples, the slope of the first normal field region became $0 \mathrm{D}$, but it has changed to quasi-2D in the mean field region.

However, appropriate values of critical fields and currents usually make the superconducting systems more suitable for investigating the thermal fluctuation effects. In turn, due to the expected fluctuation contribution just above $T_{c}$, there is not univocal consensus about the criterion for extraction of the upper critical field from transport experimental data. The behaviors of critical fields and currents against $\operatorname{Pr}$ content are shown in Fig. 6, and similar values are listed in Table 2. It is clear that
$\mathrm{B}_{\mathrm{ab}}$ is nearly more than twice as large as $\mathrm{B}_{\mathrm{c}}$ and generally decreases with $\operatorname{Pr}$ content up to $\operatorname{Pr}=0.20$, followed by an increase with higher $\operatorname{Pr}$ content up to 0.40 , but remains close to the values of $\operatorname{Pr}=0.00$. Similar behavior is observed for $\mathrm{J}(\mathrm{c})$ at $0 \mathrm{~K}$.

The hole carriers concentration $\mathrm{P}$ can be established from $\mathrm{T}_{\mathrm{c}}$ values using the following parabolic relationship [31]:

$$
\frac{T_{c x}}{T_{c}}=1-\left[82.6(P-0.16)^{2}\right]
$$

where $T_{c}$ and $T_{c x}$ are the critical temperatures for pure and doped samples, respectively. $\mathrm{P}$ is the holecarrier concentration/Cu ion. As seen in Table 2, $\mathrm{P}$ gradually increases with increasing Pr content up to $\operatorname{Pr}=0.40$, This is, of course, not consistent with the general rule of substitution $\operatorname{Pr}^{3+}\left(\operatorname{Pr}^{4+}\right) / \mathrm{Y}^{3+}$, where $P$ should decrease with Pr addition. However, most of the previous studies based on RPr:123 systems indicated that the valence state of $\operatorname{Pr}$ in $\mathrm{Y}: 123$ superconducting systems are higher than $3^{+}(3.3,3.3$ and 3.95) [32-36].

The Ginsburg number Gi, defining the order thermal fluctuations in a superconductor, is given by $[37,38]$ :

$$
G_{i}=\left[\frac{\pi \kappa^{2} \xi_{0}(c) K_{B} T_{c} \mu_{0}}{2 \phi_{0}^{2}}\right]^{2}
$$

where $\mu_{0}=4 \pi \times 10^{-7} \mathrm{~A} / \mathrm{m}$ and $\mathrm{\kappa}$ is GinsbergLandau parameter of the superconducting system given by $\lambda(0) / \xi_{c}(0)$. The values of $\mathrm{Gi}$ have been calculated at different values of Pr and listed in Table 2. It was found that $\mathrm{Gi}=7.81 \times 10^{-4}$ for $\mathrm{Y}: 123$ 
Table 2. $\mathrm{B}_{\mathrm{c}}, \mathrm{B}_{\mathrm{ab}}, \mathrm{J}_{\mathrm{c}}$ and $\mathrm{P} / \mathrm{Cu}$ for $\mathrm{Y}_{1-\mathrm{x}} \mathrm{Pr}_{\mathrm{x}}: 123$ samples.

\begin{tabular}{ccccccc}
\hline $\mathrm{x}$ & $\begin{array}{c}\mathrm{B}_{\mathrm{c}}(0) \\
{[\mathrm{T}]}\end{array}$ & $\begin{array}{c}\mathrm{B}_{\mathrm{ab}}(0) \\
{[\mathrm{T}]}\end{array}$ & $\begin{array}{c}\mathrm{J}_{\mathrm{c}}(0) \\
{\left[\mathrm{A} / \mathrm{cm}^{2}\right]}\end{array}$ & $\begin{array}{c}\mathrm{P} / \mathrm{Cu} \\
\text { ion }\end{array}$ & $\kappa$ & $\mathrm{G}_{\mathrm{i}}$ \\
\hline \hline 0.00 & 758.05 & 1090.98 & $4.18 \times 10^{4}$ & 0.160 & 326.80 & $7.81 \times 10^{4}$ \\
0.10 & 671.62 & 1044.48 & $4.08 \times 10^{4}$ & 0.192 & 331.86 & $7.13 \times 10^{4}$ \\
0.20 & 552.39 & 950.43 & $3.89 \times 10^{4}$ & 0.203 & 337.84 & $6.08 \times 10^{4}$ \\
0.30 & 542.82 & 969.38 & $3.93 \times 10^{4}$ & 0.225 & 343.25 & $3.76 \times 10^{4}$ \\
0.40 & 618.98 & 1009.71 & $4.01 \times 10^{4}$ & 0.239 & 352.94 & $2.22 \times 10^{4}$ \\
\hline
\end{tabular}

$(\operatorname{Pr}=0)$, and gradually decreased to $2.22 \times 10^{-4}$ for $\operatorname{Pr}=0.40$. These values are comparable with the reported, $G_{i}=10^{-3}$ to $10^{-4}$ for HTSC, and they are several orders of magnitude larger than $10^{-9}$ for a conventional superconductor. Therefore, the fluctuation effect has been observed in our HTSC samples and could be obtained in conventional superconductors only in forms of thin films or onedimensional wires but not in a bulk form [39, 40]. However, the present values of $\mathrm{G}_{\mathrm{i}}$ are comparable with HTSC in YPr:123 superconductors even if $\mathrm{T}_{\mathrm{c}}$ is decreased to $44.3 \mathrm{~K}$ for $\mathrm{Pr}=0.40$.

Anyhow, four different regions have been reported in the irradiated $\mathrm{Bi}: 2212$, and pure and doped Y:123 samples, respectively [41, 42]. In the present case, the 3D critical exponents were obtained in the regions very close to $\mathrm{T}_{\mathrm{c}}$ in the critical field region for $\operatorname{Pr}=0,0.10$ and 0.20 samples as well as Y:123 systems. The quasi-1D obtained for $\operatorname{Pr}=0.30$ and 0.40 may be related to decreasing the hole carriers and increasing anisotropy. The normal exponent is related to two dimensional (2D) behavior of $\operatorname{Pr}=0,0.10$ and 0.20 samples and is usually observed in the normal region of Y:123 systems. The $0 \mathrm{D}$ for $\operatorname{Pr}=0.30$ and 0.40 is also obtained. Actually, when we look at the values of OPD one can find a systematic variation between the order parameter exponents and Pr content. On the other hand, the behaviors of critical fields and currents against $\operatorname{Pr}$ content may be related to the change of order parameter from 3D to quasi-2D and system anisotropy, and therefore a direct relationship between the order parameter and critical values of the field and current could be obtained.

However, the increase in the OPD observed near the critical field region for $\operatorname{Pr}=0.30$ and 0.40 samples suggests an anomalous dimensional crossover due to suppression of superconductivity as reported in the literature [14]. A quasi-2D suggests a deterioration in the $\mathrm{CuO}_{2}$ coupling as indicated by the decreasing values of $\mathrm{J}$ and anisotropy. The 0D obtained in the mean field region may be due to oxygen rearrangement occurring in these samples rather than oxygen deficiency [14]. In addition, the strong hybridization between $(\mathrm{Pr}-4 \mathrm{f})$ and $(\mathrm{O}-2 \mathrm{P})$ conduction band electrons leading to localization of holes, offers a possibility of nonsuperconductivity in Pr:123 compounds [43, 44]. Furthermore, the STM studies based on YPr:123 single crystals show an increase in the formation of oxygen vacancies in the $\mathrm{Cu}-\mathrm{O}_{2}$ planes, where superconductivity is believed to exist. The $\operatorname{Pr}=0.00$, 0.10 and 0.20 samples look more 3D near the critical field region and 2D in the mean field region. Therefore, we do not expect decoupling of the $\mathrm{CuO}$ chains which causes an increase in $\Delta \sigma$ [45]. Hence, the behavior of these samples is nearly similar to the pure Y:123 sample and the planes remain coupled strongly.

Actually, Y:123 systems are three dimensional (3D) with two $\mathrm{Cu}-\mathrm{O}_{2}$ planes and one $\mathrm{Cu}-\mathrm{O}$ chain. The coupling between the planes and chains plays a major role in superconductivity of these materials. Therefore, the interlayer coupling of these materials is larger as compared to the BSCCO systems, and consequently, the degree of anisotropy may be lower. It has been reported that the oxygen vacancies created in $\mathrm{Cu}-\mathrm{O}_{2}$ planes can decrease the overlap between $\mathrm{Cu}-3 \mathrm{~d}$ and $\mathrm{O}-2 \mathrm{p}$ orbital, and consequently the system becomes more anisotropic, in agreement with the obtained values of $\gamma[27,46]$. Therefore, the effects of $\mathrm{Pr}$ in the overdoped 

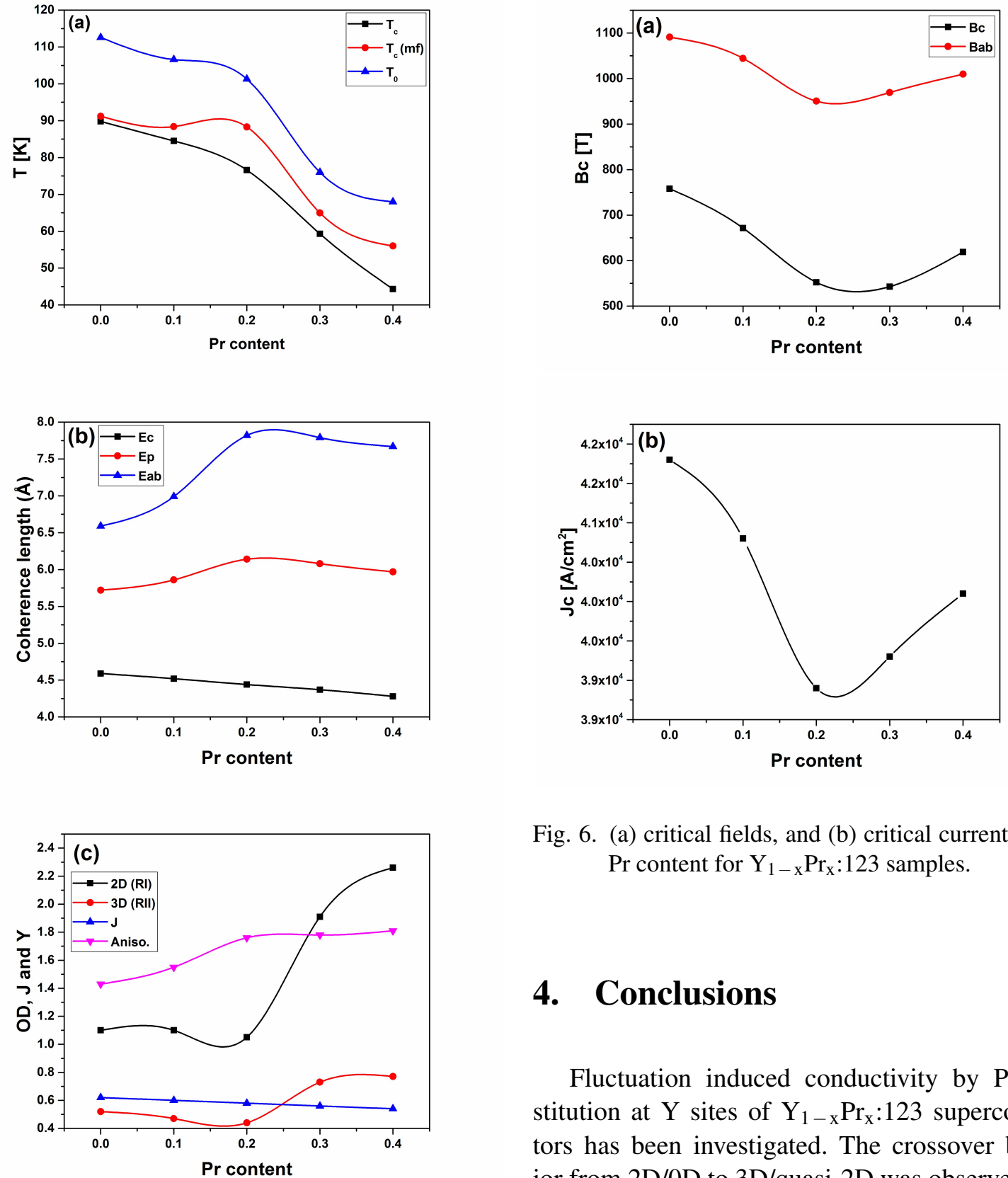

Fig. 6. (a) critical fields, and (b) critical current versus $\operatorname{Pr}$ content for $\mathrm{Y}_{1-\mathrm{x}} \operatorname{Pr}_{\mathrm{x}}$ :123 samples.

\section{Conclusions}

Fluctuation induced conductivity by $\mathrm{Pr}$ substitution at $\mathrm{Y}$ sites of $\mathrm{Y}_{1-\mathrm{x}} \operatorname{Pr}_{\mathrm{x}}: 123$ superconductors has been investigated. The crossover behavior from 2D/0D to 3D/quasi-2D was observed near $\mathrm{T}_{\mathrm{c}}$ in the investigated samples. The interlayer cou-

Fig. 5. (a) $\mathrm{T}_{\mathrm{c}}, \mathrm{T}_{\mathrm{c}}^{\mathrm{mf}}$ and $\mathrm{T}_{0}$, (b) coherence lengths, and (c) OD and $J$ versus Pr content for $\mathrm{Y}_{1-\mathrm{x}} \mathrm{Pr}_{\mathrm{x}}: 123$ samples.

pling, coherence lengths, critical fields and critical current decreased with increasing Pr up to 0.20, followed by an increase with a further increase of $\mathrm{Pr}$ up to 0.40 . However, the hole carriers/Cu ions, anisotropy, and G-L parameter gradually increased with Pr up to 0.40 . The effects of Pr in the

regions, such as oxygen rearrangements, overdoped regions such as oxygen rearrangement, anisotropy, hybridization and localization of holes may be the reason for the obtained results. anisotropy, hybridization and localization of holes may be the reason for the obtained results. 


\section{References}

[1] Cimberle M.R., Ferdeghini C., Giannini E., Marre D., Putti M., Siri A., Federici F., VArLOMOV A., Phys. Rev. B, 55 (1997), 14745.

[2] Harabor A., Harabor N.A., Deletter M., J. Optoelectron. Adv. M., 8 (2006), 1072.

[3] Mohanata A., Behera D., Physica $C, 470$ (2010), 295.

[4] Fisher D.S., Fisher M.P.A., Huse D.A., Phys. Rev. $B, 43$ (1991), 130.

[5] Aswal D.K., Singth A., SEN S., KaUR M., Viswandham C.S., Goswami G.L., Gupta S.K., J. Phys. Chem. Solids, 63 (2002), 1797.

[6] Sahoo M., Behera D., J. Phys. Chem. Solids, 74 (2013), 950

[7] Esmaeili A., Sedghi H., J. Alloy. Compd., 537 (2012), 29.

[8] Ibrahim E.M.M., SAleh S.A., Supercond. Sci. Technol., 20 (2007), 672.

[9] Fruchter L., SFAR I., Bouquet F., Li Z.Z., RAFFy H., Phys. Rev. B, 69 (2004), 144511.

[10] Baraduc C., Pagnon V., Buzdin A., Henry J., AYACHE C., Phys. Lett. A, 166 (1992), 267.

[11] GHosh A.K., Bandyopadhyay S.K., BASU A.N., Mod. Phys. Lett. B, 11 (1997), 1013.

[12] Ausloos M., Laurent Ch., Patapis S.K., PoliTis C., Lit H.L., Godelaine P.A., Gillet F., Dang A., Cloots R., Z. Phys. B, 83 (1991), 355.

[13] Konsin P., Sorkin B., Ausloos M., Supercond. Sci. Technol., 11 (1998), 1.

[14] Sedky A., Anurag G., Narlikar A.V., Phys. Status Solidi B, 241 (4) (2004), 895.

[15] Buckley R.G., Tallon J.L., Pooke D.M., PresLAND M.R., Physica C, 165 (1990), 391.

[16] Das A., Suryanarayanan R., J. Physique, 15 (1995), 623.

[17] Anderson W., Zou Z., Phys. Rev. Lett., 60 (1988), 132.

[18] Aslamazov L.G., Larkin A.I., Phys. Lett. A, 26 (1968), 238.

[19] Benazzouz F., Annabi M., Zouaoui M., BenSALEM M., Phys. Status Solidi C, 9 (2006), 2978.

[20] Ghosh A.K., BANDYOPADHYAY S.K., BASU A.N., J. Appl. Phys., 86 (1999), 3247.

[21] Sedky A., J. Low Temp. Phys., 148 (2007), 53.

[22] Ghosh A.K., Bandyopadhyay S.K., Barat P., Pintu Sen, Basu A.N., Physica C, 264 (1996), 255.

[23] Esmaeili A., Sedghi H., J. Alloy. Compd., 537 (2012), 29.

[24] Aboualy A.I., Ibrahim I.H., Awad R., ELHarizy A., Khalaf A., J. Supercond. Nov. Magn., 23 (7) (2010), 1325.

[25] Mohapatra U.K., Biswal R., Behera D., Mishra N., Supercond. Sci. Technol., 19 (2006), 635.

[26] Ramallo M.V., Torron C, Vidal F., Physica $C$, 230 (1994), 97.
[27] Ghosh A.K., Bandyopadhyay S.K., BARAT P., Pintu Sen, Basu A.N., Physica C, 255 (1995), 319.

[28] Reggiani L., Vaglio R., Varlamoa A., Phys. Rev. $B, 44$ (1991), 9541.

[29] Oh B., Char K., Kent A.D., Naito M., Beasley M.R., Geballe T.H., Hammond R.H., KapitulNik A., Graybeal J.M., Phys. Rev. B, 37 (1988), 1988.

[30] Friedmann T.A, Rice J.P., JOHn GiapintzaKis, GiInsberg D.M., Phys. Rev. B, 39 (1989), 4258.

[31] Neeleshwar N., Muralidhar M., MuRAKAmi M., Venugopal Reddy P., Physica $C$, 391 (2003), 131.

[32] Ghorbani S.R., Lundqvist P., Andersson M., Valldor M., Rapp O., Physica C, 353 (2001), 77.

[33] Lundqvist P., Grain P., Rapp O., Bryntse I., Physica C, 289 (1997), 137.

[34] Neumeier J.J, BJornholm T., Maple M.B., Rhyne J.J, Gotass J.A., Physica C, 166 (1990), 191

[35] Guillaume M., Allenspach P., Henggeler W. Mesot J., Roessli B., Staub U., Fischer P., FurRer A., Trounov V., J. Phys. Cond. Mater., 6 (1994), 7963.

[36] Obertelli S.D., Cooper J.R., Tallon J.L., Phys. Rev. B, 46 (1992), 14982.

[37] Jaraszynski J., Riggs S.C., Hunte F., GureVICH A., LARbalestier D.CbOebinger G.S., BALAKIREV F.F., Migliori A., REN Z.A., LU W., YANG J., Shen X.L., Dong X. LZHAO Z.X., Jin R. Sefat A.S., McGuire M.A., Sales B.C., ChrisTEN D.K., Mandrus D., Phys. Rev. B, 78 (2008), 064511

[38] Mun M.O., Lee S.I., LeE W.C., Phys. Rev. B, 56 (1997), 14668

[39] Pribulova Z., Kacmarcik J., Marcenat C., KoncZYKowski M., Bud'Ko S.L., Tillman M., CANFIEld P.C., Phys. Rev. B, 79 (2009) 020508.

[40] Kim S., Choi C., Jung M., Yoon J., Wang Y., Chen X., Wang X., Lee, S., Choi K., J. Appl. Phys., 108 (6) (2010), 063916.

[41] Vidal F., Veira J.A., JMaza J., Garcia F. Alvarado, Moran E., Alario M.A., J. Phys. $C, 21$ (1988), L9.

[42] Veira J.A., Maza J., Vida F.J., Phys. Lett. A, 13 (1988), 310

[43] Liechtenstein A.I., Mazen I.I., Phys. Rev. Lett., 74 (1995), 1000.

[44] Fehrenbacher R., Rice T.M., Phys. Rev. Lett., 70 (1993), 3471.

[45] Rusiecki S., Bucher B., Kaldis E., JileK E., KARPINSKi J., J. Less-Comm. Met., 164 - 165 (1990), 31.

[46] Mandal P., Poddar A., Das S., J. Phys. Condens. Mater., 6 (1994), 5689

Received 2017-05-05 Accepted 2018-01-17 